\title{
Europa tra storia e futuro nella prospettiva dei diritti umani
}

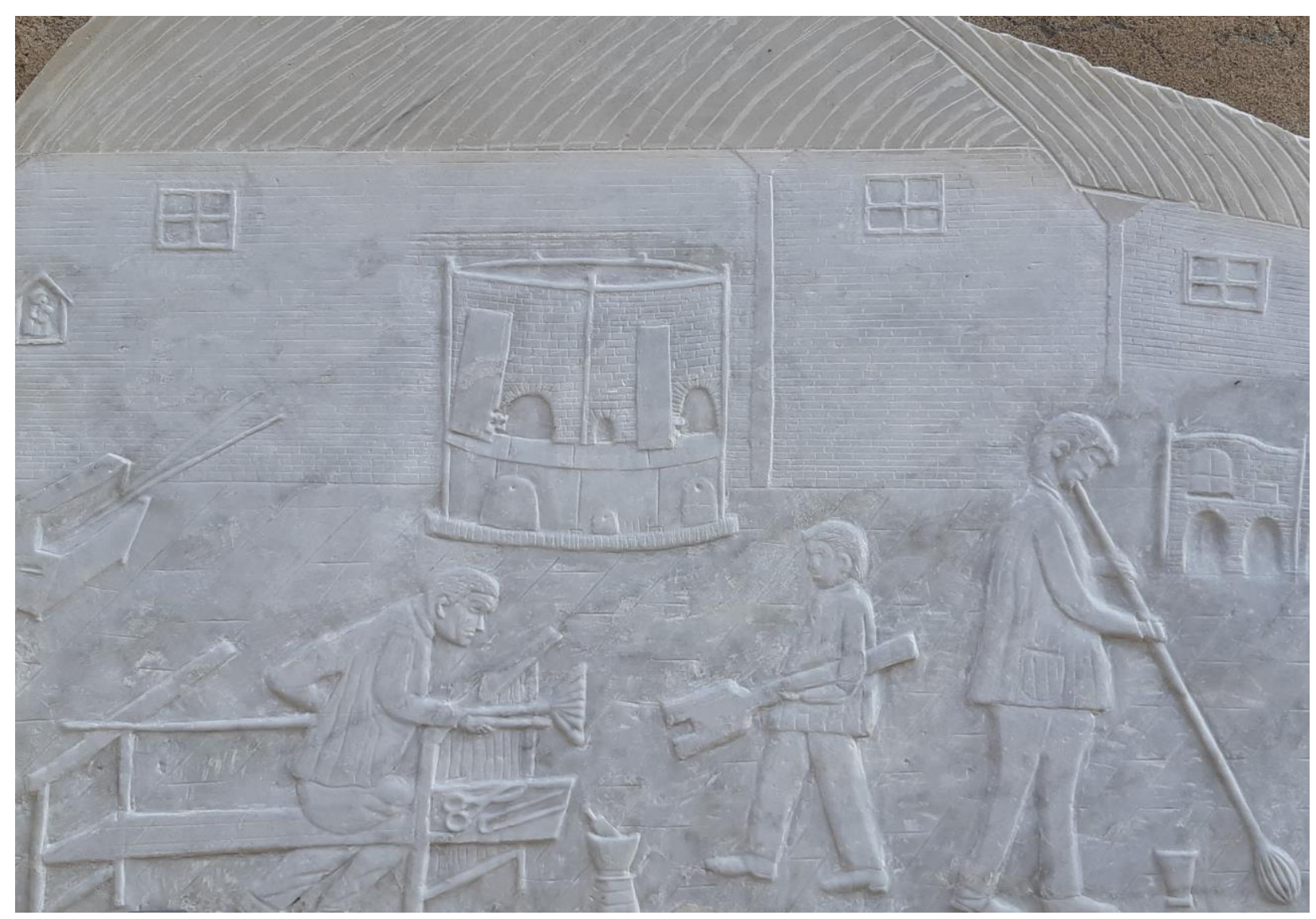

2021 ANNO VI NUMERO 12

di Marco A. Quiroz Vitale e https://doi.org/10.54103/2531-6710/17375: ... 


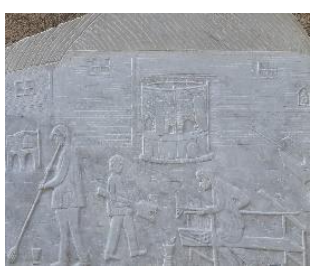

Società e diritti - rivista elettronica anno 2021, V n. 12

\section{EUROPA TRA STORIA E FUTURO NELLA PROSPETTIVA DEI DIRITTI UMANI}

\section{di Marco A. Quiroz Vitale}

La Rivista SeD, Società e diritti, presenta in questo numero contributi multidisciplinari dedicati alla cultura europea oltre ad altri saggi ispirati ai problemi emergenti della realtà contemporanea.

Il numero si apre con un illuminante saggio del filosofo del diritto Francesco Petrillo che richiama alla necessità di ricostituire un tessuto comune per l'Europa, a partire dal riconoscimento di natura sostanziale dei diritti individuali e del loro fondamento, rintracciabile proprio nella tradizione della Res publica chistiana che ha visto il sorgere dell'idea stessa d'Europa come luogo della pratica della giustizia universale e del rispetto dei diritti umani. Secondo l'Autore, e noi sottoscriviamo l'affermazione, nessuna alleanza economica, finanziaria o giuridico-costituzionale può permettere, oggi, un rilancio dell'Europa unita, quanto quella che faccia propria la prospettiva dei diritti umani fondamentali.

Sulla stessa linea di pensiero, ma in termini più severi e critici, si pone il secondo saggio del professore Alessandro Catelani che, con una più sintetico ma non meno pungente contributo, osserva come l'Impero carolingio (dalla notte di natale dell' 800 d.C. sino alla deposizione di Carlo il Grosso) e poi il Sacro Romano Impero per circa mille anni, sia stata l'unica attuazione effettiva dell'idea d'Europa, resa ora ardua dalla rischiosa eliminazione degli Stati nazionali con le garanzie di diritto e giustizia che rappresentano tuttora. Il problema così sollevato della difficoltà di "fondere tutti i popoli di un intero continente in unico Stato" non solo è certamente reale ma è aggravato dalla assenza di una chiara dimensione costituzionale, federale o statuale, che caratterizzi l'Unione; come lascia intendere anche Petrillo è stata costituita l'Europa ma non si sono fatti gli europei. I due saggi che aprono il numero di SeD pur scritti a partire da fronti contrapposti 
appaiono singolarmente complementari nella compressione del problema europeo del terzo millennio dell'era cristiana.

Alla luce del dibattito delineato supra, assume una dimensione diversa ed ancor più preoccupante il terzo saggio della dott.ssa Anna Marotta che, affrontando con attenzione e profondità, il tema dell'operatività delle Corti islamiche in Inghilterra, mostra le contraddizioni in cui si dibatte il Vecchio Continente tra il naufragio dell'illusione della multiculturalità e l'attuale fascinazione per la intercultura. Le conclusioni dell'Autrice secondo cui si potrebbe ricostruire un percorso storico contraddistinto dalle politiche dei governi occidentali volte a far spazio a tutte le diversità alla luce dei valori democratici e liberali sono da ripensare con attenzione. Il caso inglese, che ha manifestato progressive aperture del diritto positivo alla pluralità culturale mediante una politica delle 'differenze', dando luogo a inevitabili conflitti e contraddizioni, è certamente emblematico; tuttavia la pericolosità di tale crinale è ancor più evidente nella fragile Europa priva di una common law o di una architettura istituzionale adeguata. Il riferimento ai diritti umani per dirimere simili scontri pare arduo in quanto in essi sempre meno può trovarsi una regola per la soluzione dei conflitti culturali e delle liti individuali e sempre più gli stessi Human Rights paiono divenire strumenti per attuare scontri ideologici e materiali in un Continente che rischia di abdicare alle proprie radici culturali e valoriali. Una ventata di novità ed interesse giungono anche dagli due saggi del prof. Munguia Salazar e dei dottori Mazzola e Rancati dedicati ai diritti umani in una prospettiva internazionale, il primo saggio incentrato sulla violazione dei diritti fondamentali nello scontro USA-Messico ed il secondo ispirato dalla prospettiva del mercato del sesso in Tailandia. Il tema dei diritti umani costituisce un motivo dominante di interesse per la rivista $\mathrm{SeD}$, tuttavia, entrambi i saggi pubblicati suscitano la necessità di un ripensamento profondo delle tecniche di analisi del fenomeno giuridico, in una ottica realista, soprattutto nel campo dei diritti che sorgono e si affermano "prima" ed "al di sopra" delle legislazioni nazionali. Soccorre con tutta evidenza il magistrale saggio del dott. Enrico Damini che affronta con competenza e profondità il tema dell'efficacia del diritto a partire dalla dottrina di Theodor Geiger. Chiude il numero di SeD l'originale e attualissimo contributo dei docenti dell'Università Statale di Milano (Paola Parravicini e Alessandro Graffi), dedicato alla formazione professionale ed alle variabili sociologiche che caratterizzano la futura classe docente delle scuole secondarie di I e di II grado del nostro Paese.

Il Direttore 